\title{
The expression of the ubiquitin ligase SIAH2 (seven in absentia homolog 2) is mediated through gene copy number in breast cancer and is associated with a basal-like phenotype and p53 expression
}

Peter Chan ${ }^{1 \dagger}$, Andreas Möller ${ }^{2,3^{*}+}$, Mira CP Liư ${ }^{2}$, Jaclyn E Sceneay ${ }^{2}$, Christina SF Wong ${ }^{2}$, Nic Waddell ${ }^{4}$, Katie T Huang 1,3, Alexander Dobrovic ${ }^{1,3}$, Ewan KA Millar ${ }^{5,6,10,11}$, Sandra A O'Toole ${ }^{7,12}$, Catriona M McNeil ${ }^{8,9}$, Robert L Sutherland ${ }^{5}$, David D Bowtell ${ }^{2}$, Stephen B Fox ${ }^{1,3^{*}}$

\begin{abstract}
Introduction: The seven in absentia homolog 2 (SIAH2) protein plays a significant role in the hypoxic response by regulating the abundance of hypoxia-inducible factor- $\alpha$; however, its role in breast carcinoma is unclear. We investigated the frequency and expression pattern of SIAH2 in two independent cohorts of sporadic breast cancers.

Methods: Immunohistochemical evaluation of SIAH2protein expression was conducted in normal breast tissues and in tissue microarrays comprising ductal carcinoma in situ (DCIS) and a cohort of invasive breast carcinomas. Correlation analysis was performed between $\mathrm{SIAH} 2$ and clinicopathological variables and intrinsic breast cancer subgroups and validated in a cohort of 293 invasive ductal carcinomas. Promoter methylation, gene copy number and mRNA expression of SIAH2 were determined in a panel of basal-like tumors and cell lines.
\end{abstract}

Results: There was a significant increase in nuclear SIAH2 expression from normal breast tissues through to DCIS and progression to invasive cancers. A significant inverse correlation was apparent between $\mathrm{SIAH} 2$ and estrogen receptor and progesterone receptor and a positive association with tumor grade, HER2, p53 and an intrinsic basal-like subtype. Logistic regression analysis confirmed the significant positive association between SIAH2 expression and the basal-like phenotype. No SIAH2 promoter methylation was identified, yet there was a significant correlation between SIAH2 mRNA and gene copy number. SIAH2-positive tumors were associated with a shorter relapse-free survival in univariate but not multivariate analysis.

Conclusions: $\mathrm{SIAH} 2$ expression is upregulated in basal-like breast cancers via copy number changes and/or transcriptional activation by p53 and is likely to be partly responsible for the enhanced hypoxic drive through abrogation of the prolyl hydroxylases.

\section{Introduction}

Hypoxia in breast cancer has profound effects on tumor biology that are reflected in a poor prognosis and resistance to both chemotherapy and radiotherapy in patients [1]. Hypoxia-inducible factor (HIF)-1 is critical to the hypoxic response, being a transcription factor that,

\footnotetext{
* Correspondence: andreas.moeller@petermac.org; stephen.fox@petermac.org † Contributed equally

'Department of Pathology, Peter MacCallum Cancer Centre, Locked Bag 1 A'Beckett Street, Melbourne, Victoria 8006, Australia

${ }^{2}$ Research Division, Cancer Genetics and Genomics, Peter MacCallum Cancer Centre, Locked Bag 1 A'Beckett Street, Melbourne, Victoria 8006, Australia Full list of author information is available at the end of the article
}

through binding to hypoxia response elements in the promoters of genes, results in expression of proteins involved in angiogenesis (vascular endothelial growth factor (VEGF)), glucose metabolism (glucose transporter 1), metastasis (chemokine (C-X-C motif) receptor 4 and stromal cell-derived factor-1), cell survival and proliferation.

HIF-1 is a dimer consisting of a constitutively expressed aryl nuclear translocator or HIF- $1 \beta$ and a hypoxia-inducible HIF-1 $\alpha$. The levels of HIF- $1 \alpha$ are tightly regulated by three prolyl hydroxylases. In the presence of molecular oxygen, these enzymes hydroxylate the prolyl residues 402 and 564 in the oxygen-dependent 
domain of HIF-1 $\alpha$, resulting in conformational change and recognition by the von Hippel-Lindau protein that leads to its ubiquitination and degradation via the proteasome. In contrast, under hypoxia, the prolyl hydroxylases have limited molecular oxygen and are therefore less effective, which enables HIF- $1 \alpha$ stabilization, translocation to the nucleus and initiation of gene transcription that benefits the tumor.

Seven in absentia homolog 2 (SIAH2) is one of a family of RING domain proteins which act alone or as components of ubiquitin ligase complexes that target proteins for proteasomal degradation [2]. Siah proteins can interact with many intracellular pathways, including the scaffold proteins, transcriptional repressors and nuclear receptor corepressors and $\beta$-catenin. Siah proteins are also involved in hypoxia signaling via regulation of HIF-1 $\alpha$ [3] through the targeted degradation of prolyl hydroxylases under hypoxic conditions. Indeed, SIAH2-knockout mice have a delayed and abrogated response to hypoxic conditions that is mediated through reduced levels of HIF-1 $\alpha[3,4]$. These data suggest that Siah proteins may significantly alter HIF signaling through modulation of the prolyl hydroxylases.

Although the role of HIF has been documented in breast cancer $[5,6]$, there are no data on the expression of SIAH2 in this disease. We have therefore investigated SIAH2 expression in breast cancer in two independent cohorts. Our aims were to (1) document the pattern and level of SIAH2 expression in breast cancer, (2) correlate expression with conventional clinicopathological factors, (3) investigate associations of SIAH2 expression with intrinsic subtypes of breast cancer and (4) determine the effect of SIAH2 expression on relapse-free survival.

\section{Materials and methods \\ Patients}

The flow of patients through the study according to the Reporting Recommendations for Tumor Marker Prognostic Studies (REMARK) criteria is listed in Supplementary Table 1 in Additional file 1 [7] The first cohort was derived from the Department of Pathology, Peter MacCallum Cancer Centre, Melbourne, Australia, and comprised 120 cases with full clinicopathological characteristics but without survival data. The second cohort was from the Garvan Institute, Sydney, Australia, and comprised 293 cases with full clinicopathological characteristics including survival data [7]. In total, 439 invasive cancers with clinicopathological data and follow-up were available for study. Of these 439 cases, 61 cases were excluded because of inadequate tumor tissue on the array. The final cohort of invasive cancers comprised 378 cases (246 cases with survival data). Eighty cases of pure ductal carcinoma in situ (DCIS) were obtained from the John Radcliffe Hospital, Oxford, UK, of which 54 had DCIS on tissue microarrays (TMAs) for staining and clinical data available. Ten cases of normal postmenopausal breast tissues from mammoplasties were also collected. This study has Ethics Committee approvals (numbers 00/81, 03/90, 09/36, JRC02.216, HREC SVH H94/080 and H00/36). All patients had operable breast carcinomas and were not diagnosed with distant metastatic disease at the time of presentation. Information regarding patient characteristics, including age, tumor size, grade, histology and nodal status were collected from the clinical and pathological records. The median age of patients included in this study was 54 years (range, 24 to 87 years). Ninety-three percent of tumors were invasive ductal tumors not otherwise specified type, $3 \%$ were invasive lobular carcinomas and $4 \%$ were of other histological types (data were unavailable for two cases). Median tumor size was $20 \mathrm{~mm}$, and the median tumor grade was 2. Forty-one percent of patients had nodal disease. Sixty-nine percent of tumors were estrogen receptor (ER)-positive, and 14\% were human epidermal growth factor receptor 2 (HER2)-positive. Patients less than 50 years of age with node-positive, ER-negative tumors or tumors larger than $3 \mathrm{~cm}$ received adjuvant chemotherapy (cyclophosphamide, methotrexate and 5-fluorouracil or adriamycin and cyclophosphamide. Patients with hormone-responsive tumors who were more than 50 years of age received 5 years of endocrine therapy. Patients were followed up for a median period of 58.1 months. During this time, in the 100 patients from the invasive cohort, two developed recurrence and 86 deaths were considered breast cancer-related.

\section{Immunohistochemistry}

TMAs were constructed from 1-mm diameter (invasive cancers) or 2-mm cores (DCIS). Sections of 4- $\mu \mathrm{m}$ thickness were used for immunostaining. TMA sections were dewaxed, and antigen retrieval was performed in $10 \mathrm{mM}$ sodium citrate, $\mathrm{pH} 6$, in a pressure cooker for $3 \mathrm{~min}$ utes. Sections were then treated with $3 \% \mathrm{H}_{2} \mathrm{O}_{2}$ for 5 minutes to remove endogenous peroxides, washed and incubated with a SIAH2 antibody (NB110-88113; Novus Biologicals, Littleton, CO, USA) $[9,10]$ at 1:50 dilution for 90 minutes at room temperature. The peroxidasecoupled Mouse ImmPRESS (Vector Laboratories, Burlingame, CA, USA) detection reagent was then used, and staining was visualized with diaminobenzidine plus (DAB+; Dako, Campbellfield, VIC, Australia). Sections were counterstained with hematoxylin to visualize nuclei. To analyze the expression of SIAH2 in breast cancer progression, we assessed expression using a combination of both intensity and proportion of cells expressing SIAH2 [10] Normal breast epithelium and tumors were scored for intensity $(0=$ no staining, 
$1=$ weak staining, $2=$ moderate staining and $3=$ strong staining) and the percentage of cells $(0=$ no cells staining positive, $1=<10 \%$ cells staining positive, $2=10 \%$ to $50 \%$ cells staining positive, $3=51 \%$ to $80 \%$ cells staining positive and $4=>80 \%$ cells staining positive) as previously reported [12]. The scores for intensity and percentage of positive tumor cells were added to give a maximum score of 7 . A cutoff of $>2$ (median value) was used to define two patient groups of approximately equal size for subsequent statistical analyses.

ER, HER2, epidermal growth factor receptor (EGFR) and cytokeratin (CK)5/6 staining were used to classify tumors into four intrinsic subgroups: the basal group (ER-negative, HER2-negative, CK5/6-positive and/or EGFR-positive), the luminal group (ER-positive, HER2negative), the HER2 group (HER2-positive) and the negative (null) group (ER-negative, HER2-negative, CK5/ 6-negative and EGFR-negative) [13].

\section{Analysis of SIAH2 Methylation}

DNA from a separate series of 60 breast carcinomas (John Radcliffe Hospital, Oxford, UK) and five normal breast tissues (Peter MacCallum Cancer Centre, Melbourne, VIC, Australia), comprising all breast cancer phenotypes (50 ER-positive and 10 ER-negative) (also see Supplementary Table 2 in Additional file 2), and DNA was also obtained from the breast cancer cell lines MCF-10A, MCF-7, BT20, SkBr3, Hs578T, T47D, MDAMB-157, MDA-MB-468, MDA-MB-453, MDA-MB-231, MDA-MB-361, BT483 and ZR75. Bisulfite-modified (EpiTect Bisulfite kit; Qiagen, Hilden, Germany) DNA were assessed for SIAH2 methylation using methylationsensitive high-resolution melting (MS-HRM) [14]. The MS-HRM primers for SIAH2 were as follows: 5'-TAGAAGCGGGTGGGTTAGGGTTT-3' (forward) and 5'-CTAATACACTCCGCAACCCCC-3' (reverse) amplified a region corresponding to GenBank accession number AC011317.23, nucleotides 105279 to 105409, which contains $17 \mathrm{CpG}$ islands. A polymerase chain reaction (PCR) assay was performed in a final volume of $20 \mu \mathrm{l}$. The PCR reaction mixture consisted of $1 \times$ PCR buffer (Qiagen), $2.5 \mathrm{mM} \mathrm{MgCl} 2,200 \mu \mathrm{M}$ concentrations of each deoxyribonucleotide triphosphate, a $200 \mathrm{nM}$ concentration of the forward primer, a $200 \mathrm{nM}$ concentration of the reverse primer, $5 \mu \mathrm{M}$ SYTO9 intercalating dye (Invitrogen, Mulgrave, VIC, Australia), 0.5 U of HotStarTaq DNA Polymerase (Qiagen), and $1 \mu \mathrm{l}$ (theoretical amount $10 \mathrm{ng}$ ) of bisulfite-modified DNA. The PCR amplification was performed with an activation step of 15 minutes at $95^{\circ} \mathrm{C}$, followed by 50 cycles of 10 seconds at $95^{\circ} \mathrm{C}, 10 \mathrm{sec}-$ onds at an annealing temperature of $66^{\circ} \mathrm{C}, 20$ seconds at $72^{\circ} \mathrm{C}$ for extension and one denaturation step of 1 minute at $97^{\circ} \mathrm{C}$. HRM was directly performed after PCR amplification. PCR products were denatured at $97^{\circ} \mathrm{C}$ for 1 minute, then cooled to $75^{\circ} \mathrm{C}$ with temperature rising by $0.2^{\circ} \mathrm{C}$ per second to $97^{\circ} \mathrm{C}$ and holding for 1 second after each stepwise increment. Methylated sequences could be identified by their increased melting temperature [13]. In each assay, fully methylated, peripheral blood DNA (unmethylated), different methylation percentage dilution standards and nontemplate controls were included as controls and standards. All assays were performed in duplicate.

\section{Copy number analysis of SIAH2}

A previous study analyzed both gene expression and copy number variation using the Illumina Human-6 BeadArray (Illumina, San Diego, CA, 92121, USA) and the CNV370 SNP array (Illumina) respectively, in a cohort of familial tumors [14]. These familial tumors were known to be breast cancer 1 , early onset gene (BRCA1), BRCA2 or non-BRCA1 and non-BRCA2 tumors, and in this previous study the familial tumors were classified into one of the breast tumor subtypes: basal-like, luminal A, luminal B, HER2-positive and normal-like. These data were used to determine the expression of SIAH2 and its copy number status in 15 basal-like tumors. The copy number of SIAH2 in each tumor was inferred from the average $\log \mathrm{R}$ value of eight single-nucleotide polymorphisms (SNPs), which were within the SIAH2 open reading frame $(n=3)$ or in the sequence flanking the gene $(n=5)$.

\section{Results}

SIAH2 expression in normal breast, in situ and invasive breast carcinomas

SIAH2 expression was identified in the nuclei of occasional cells within the luminal layer of ducts and acini in normal breast tissues in three of 10 patients (30\%). This expression was usually of mild to moderate intensity, and when stratified using the cutoff used for the tumors, all were considered negative for SIAH2 (Figure 1A). Expression of SIAH2 was observed in the nuclei of seven of 54 (13\%) DCIS cases. The staining was generally of a moderate to strong intensity with a homogeneous distribution (Figures $1 \mathrm{~B}$ and $1 \mathrm{C}$ ). There was a nonsignificant increase in SIAH2 from the transition of normal to in situ disease $(P=0.13)$ and a significant increase in SIAH2 from in situ to invasive breast carcinoma $(P=0.0006)$ (Table 1 and Figures $1 \mathrm{D}$ and 2$)$.

\section{Association between SIAH2 protein expression and clinicopathological characteristics in DCIS}

There was no significant correlation between SIAH2 expression and nuclear grade, presence of necrosis, age, ER, progesterone receptor (PR), EGFR or HER2 (all $P>0.05$ ) or intrinsic phenotypes in DCIS $(P=0.471)$ (Supplementary Table 3 in Additional file 3). 


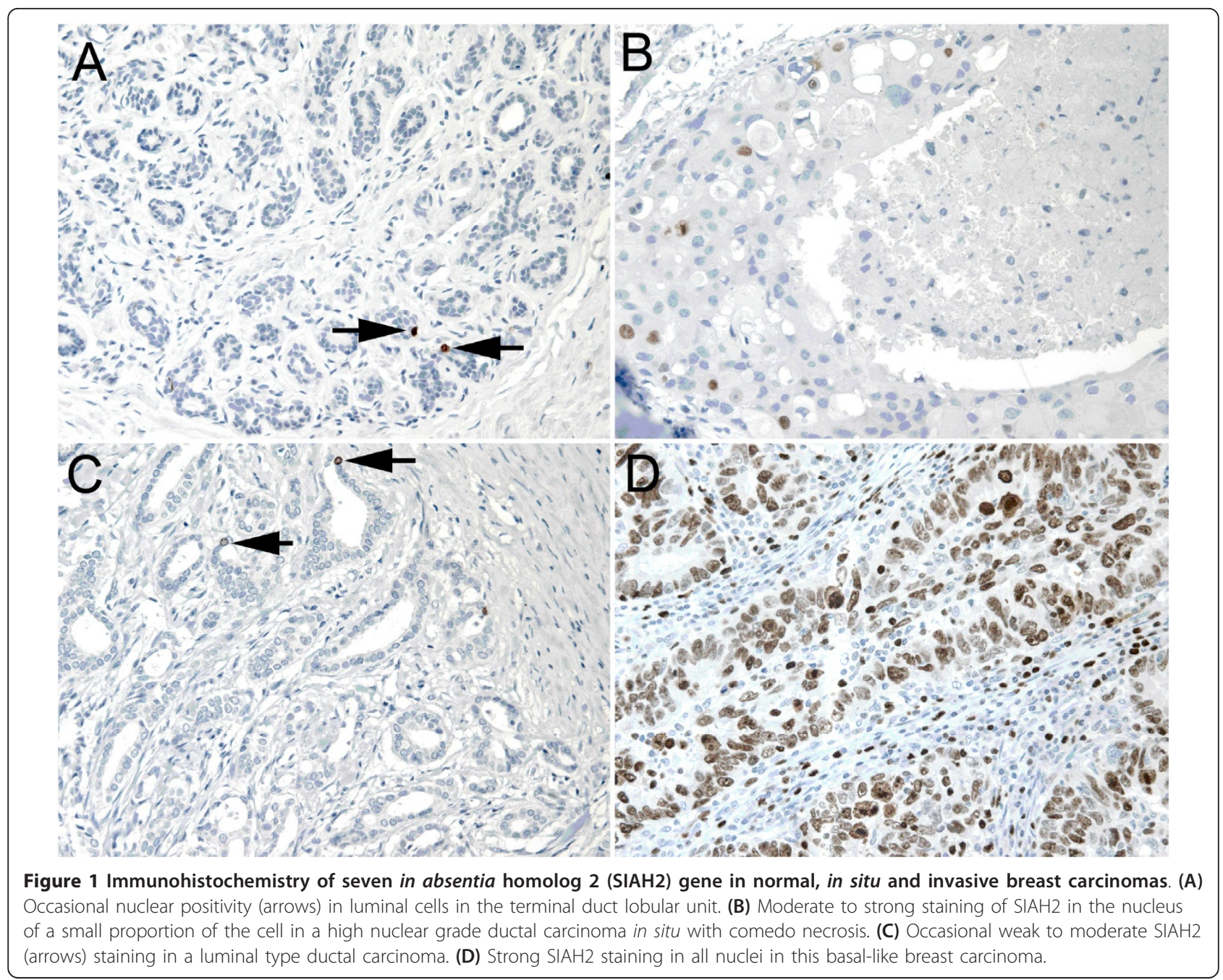

\section{Correlation between SIAH2 protein expression with} clinicopathological characteristics and intrinsic subtypes in invasive cancer

In the primary cohort, there was a significant inverse correlation between SIAH2 protein expression and ER $(P<$ $0.0001)$, PR $(P=0.011)$ and a positive association with tumor grade $(P<0.0001)$ and intrinsic subtype $(P=0.028)$, but there was no association with patient age, tumor size, lymph node status or HER2 (all $P>0.05$ ) (Supplementary

Table $1 \chi^{2}$ tests, SIAH2 expression in normal breast, DCIS and invasive cancers ${ }^{a}$

\begin{tabular}{lccc}
\hline Tissue type & Negative, $\boldsymbol{n}(\%)$ & Positive, $\boldsymbol{n}(\%)$ & Total, $\boldsymbol{n}(\%)$ \\
\hline Normal & $10(100 \%)$ & $0(0 \%)$ & $10(100 \%)$ \\
DCIS & $47(87 \%)$ & $7(13 \%)$ & $54(100 \%)$ \\
Invasive & $155(45 \%)$ & $194(55 \%)$ & $349(100 \%)$ \\
Total & $212(51 \%)$ & $201(49 \%)$ & $413(100 \%)$ \\
\hline
\end{tabular}

${ }^{a}$ SIAH2, seven in absentia homolog 2 gene; DCIS, ductal carcinoma in situ; $P<$ 0.0001 .
Table 4 in Additional file 4). In the validation cohort, there was a significant inverse correlation between SIAH2, ER $(P<0.0001)$ and PR $(P<0.0001)$ and a significant positive association with tumor grade $(P<0.0001)$, patient age $(P=$ 0.009), HER2 $(P=0.007)$ and intrinsic subtype $(P<0.0001)$, but not with tumor size or lymph node status $(P>0.05)$ (Supplementary Table 5 in Additional file 5). In the combined cohort, there was a significant inverse correlation between SIAH2 and ER $(P<0.0001)$ and PR $(P<0.0001)$ and a positive correlation with tumor grade $(P<0.0001)$, HER2 $(P=0.007)$, p53 $(P<0.001)$ and intrinsic subtype $(P<0.0001)$, but not with patient age, tumor size or lymph node status $(P>0.05)$ (Table 2$)$. The significant associations between SIAH2 expression grade and intrinsic subgroups were confirmed in a multivariate analysis of tumor phenotype, age, grade and lymph node status with the basal-like phenotype being more than five times more likely to express SIAH2 than luminal tumors (Table 3) $(P=0.015)$, which was also observed in the combined cohort $(P=0.042)$. 


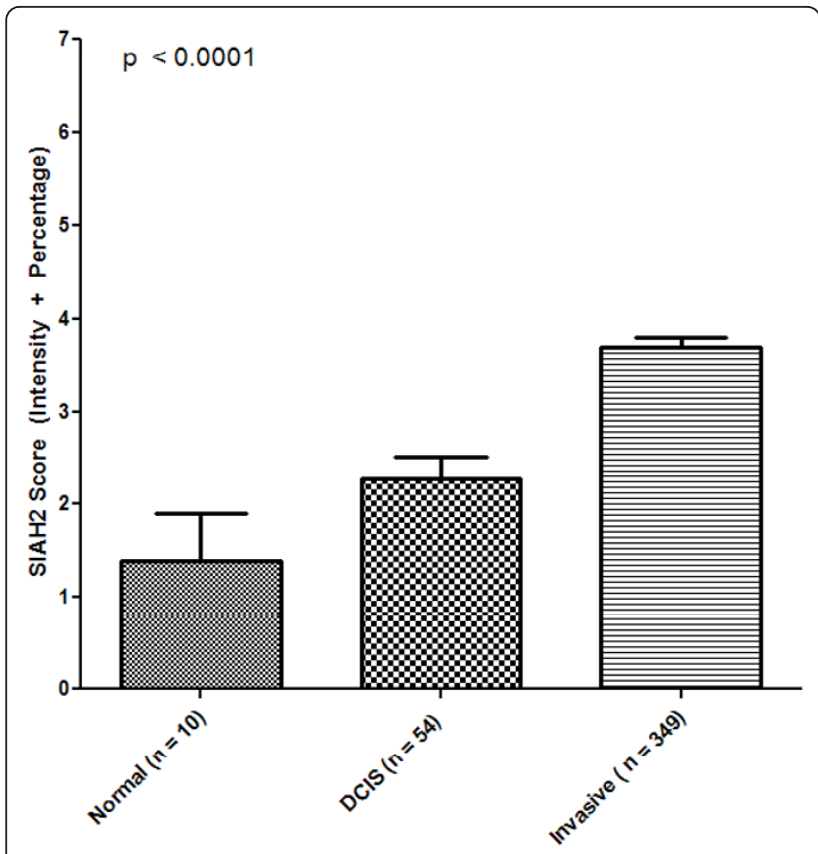

Figure 2 Semiquantitative seven in absentia homolog 2 (SIAH2) gene expression in normal, ductal carcinoma in situ (DCIS) and invasive carcinoma samples.

Promoter methylation of SIAH2 in cell lines and tumors To assess whether SIAH2 expression in tumors is modulated by promoter methylation, $\mathrm{CpG}$ islands were identified in the promoter region of SIAH2, and MSHRM primers were designed to cover the CpG-rich area of the promoter region of SIAH2. Five normal breast tissues, 60 breast carcinomas and 13 breast cancer cell lines were screened for methylation of SIAH2, but no promoter methylation was detected in these cancer cell lines and samples, as shown by the absence of altered methylation profiles (Supplementary Figure 1 in Additional file 6).

\section{Correlation of gene expression and relative copy number of $\mathrm{SIAH} 2$ in basal-like tumors}

A cohort of familial tumors, which included 15 basallike tumors, was previously analyzed on the basis of gene expression and copy number analysis [15]. The 15 basal-like tumors showed a significant correlation between SIAH2 expression and estimated copy number ( $r=0.675, P=0.003$ ) (Figure 3 ). Two of the 15 basallike tumors showed a copy number gain (copy number of 3) and a further three of 15 basal-like tumors showed loss of heterozygosity at this region. In contrast, 15 nonbasal-like tumors (10 luminal A, four luminal B and one normal-like tumor) did not show any copy number change in this region.
Relationship between SIAH2 expression and relapse-free and overall survival

There was no correlation present between SIAH2 expression and overall relapse-free survival in DCIS-only patients $(P=0.68)$. Although there was a significantly shorter relapse-free survival in all patients with invasive carcinomas stratified by SIAH2 $(P=0.002)$ (Figure 4$)$, no significant association with relapse-free survival was observed in univariate analysis in different breast cancer intrinsic groups stratified by SIAH2 (data not shown). There was also no significant association between SIAH2 in invasive carcinomas of all patients and relapse-free survival in multivariate analysis (Table 4).

\section{Discussion}

Hypoxia is a pivotal driver in breast tumor progression, leading to transcription of several suites of genes involved in angiogenesis, cell survival, cell proliferation and an enhanced metastatic phenotype that are advantageous to the neoplastic cells [1]. SIAH2 is part of the ubiquitin ligase complex that target proteins for proteasomal degradation and enhances HIF-1 $\alpha$ expression by reducing the abundance of the prolyl hydroxylases $[16,17]$. These enzymes, in the absence of SIAH2, hydroxylate prolyl residues in the oxygen-dependent domain of HIF- $1 \alpha$, resulting in HIF- $1 \alpha$ proteasomal degradation and attenuation of the hypoxic response. Since SIAH2 has the potential to profoundly influence the hypoxic response, we investigated its expression in normal and neoplastic breast tissues.

We observed significant upregulation of SIAH2 in the nucleus in the transition from normal to in situ and invasive carcinomas in breast cancer, supporting the notion of an important role of SIAH2 in breast cancer progression. SIAH2 has a nuclear localization signal that could account for its subcellular pattern of expression [18]. The increase in SIAH2 in in situ and invasive carcinomas correlates with the hypoxia that occurs in neoplasia as the metabolic demand of the tumor exceeds the supply of nutrients and oxygen from the disordered vasculature that is developing.

Correlation analysis showed a significant relationship between high levels of breast tumor SIAH2, negative ER and PR and high HER2. The absence of a positive correlation with ER is of interest, since estrogen has been reported to induce expression of SIAH2 in ER-positive breast cancer cell lines [19] and there is a positive relationship between Siah2 and ER-positive breast tumors but not the basal-like phenotype in six publicly accessible data sets [7,20-25] (analysis not shown). This discrepancy is likely due to the well-described differences between gene expression and protein abundance or to the fact that ER expression may be heterogeneous in 
Table 2 Contingency table of SIAH2 expression in invasive breast carcinomas of the combined cohort with clinicopathological parameters

\begin{tabular}{|c|c|c|c|c|}
\hline Parameter & Negative, $N=169$ (45\%) & Positive, $N=209$ (55\%) & Total, $N=378(100 \%)$ & $P$ value \\
\hline Grade & & & & $<0.0001$ \\
\hline Low & $40(78.4 \%)$ & $11(21.6 \%)$ & $51(100 \%)$ & \\
\hline Intermediate & $77(60.2 \%)$ & $51(39.8 \%)$ & $128(100 \%)$ & \\
\hline High & $32(20.4 \%)$ & $125(79.6 \%)$ & 157 (100\%) & \\
\hline Age, yr & & & & 0.187 \\
\hline$<50$ & 48 (39.3\%) & $74(60.7 \%)$ & $122(100 \%)$ & \\
\hline$>50$ & $107(47.8 \%)$ & $117(52.1 \%)$ & $224(100 \%)$ & \\
\hline Tumor size & & & & 0.168 \\
\hline$<20 \mathrm{~mm}$ & $89(48.6 \%)$ & $94(51.4 \%)$ & $183(100 \%)$ & \\
\hline$>20 \mathrm{~mm}$ & $61(39.9 \%)$ & $92(60.1 \%)$ & $153(100 \%)$ & \\
\hline Lymph node status & & & & 0.915 \\
\hline Negative & $83(45.4 \%)$ & $100(54.6 \%)$ & 147 (100\%) & \\
\hline Positive & $64(43.0 \%)$ & $85(57.0 \%)$ & $185(100 \%)$ & \\
\hline ER status & & & & $<0.0001$ \\
\hline Negative & $21(20.8 \%)$ & $80(79.2 \%)$ & $101(100 \%)$ & \\
\hline Positive & $129(54.4 \%)$ & $108(45.6 \%)$ & $237(100 \%)$ & \\
\hline PR status & & & & $<0.0001$ \\
\hline Negative & $39(28.5 \%)$ & $98(71.5 \%)$ & 137 (100\%) & \\
\hline Positive & $111(55.2 \%)$ & $90(44.8 \%)$ & $201(100 \%)$ & \\
\hline HER2 status & & & & 0.003 \\
\hline Negative & $128(48.3 \%)$ & $137(51.7 \%)$ & $265(100 \%)$ & \\
\hline Positive & $22(29.7 \%)$ & $52(70.3 \%)$ & $74(100 \%)$ & \\
\hline Tumor subtype & & & & $<0.0001$ \\
\hline Luminal & $114(57.6 \%)$ & $84(42.4 \%)$ & 198 (100\%) & \\
\hline Basal-like & $4(9.6 \%)$ & 37 (90.2\%) & 41 (100\%) & \\
\hline Her2 & $22(29.3 \%)$ & $53(70.7 \%)$ & 75 (100\%) & \\
\hline Null & 11 (44\%) & $14(56 \%)$ & 25 (100\%) & \\
\hline \multicolumn{5}{|l|}{ P53 } \\
\hline Negative & $108(57.8 \%)$ & 79 (42.2\%) & 187 (100\%) & $<0.001$ \\
\hline Positive & 12 (18.2\%) & $54(81.8 \%)$ & 66 (100\%) & \\
\hline
\end{tabular}

${ }^{a} \mathrm{SIAH} 2$, seven in absentia homolog 2 gene.

ER, estrogen receptor; HER2, human epidermal growth factor receptor 2; PR, progesterone receptor.

both the pattern and level of expression within tumors. Nevertheless, although there was an inverse relationship between SIAH2 and ER, approximately half of SIAH2positive samples expressed ER, suggesting a complex relationship.

The finding that SIAH2 was significantly associated with HER2 and basal-like intrinsic breast cancer subtypes, which for basal-like cancers was confirmed in multivariate analysis, is in accord with our previous report of an enhanced hypoxic drive in basal-like cancers [26]. In this study, we have demonstrated that basal-like breast cancers have an intrinsically elevated SIAH2 level as part of its phenotype that may, partly at least, explain the mechanism underlying high HIF-1 $\alpha$ expression in this tumor subtype. The upregulation of SIAH2 may be regulated at several levels. We investigated the potential role that $p 53$ may play, since this gene is frequently mutated in this tumor type [11]. In support of this notion is the significant correlation between SIAH2 and p53 immunostaining. A further mechanism in basal-like cancer may also involve p38 mitogen-activated protein kinase, which is also upregulated in the basal-like phenotype, as activated p38 increases the activity of SIAH2 [11]. We also explored the role of SIAH2 promoter methylation to assess whether protein expression is epigenetically repressed. We observed no evidence of methylation in any breast carcinoma cell line, normal breast or in a series of 60 breast cancers of variable phenotypes, making this mechanism of repression highly unlikely in breast tissues in either normal tissue or tumoral tissue.

We then hypothesized that since SIAH2 is located on 3q25.1, overexpression might be mediated through gene amplification. Indeed, this locus is frequently amplified in basal-like breast cancer [27], and our preliminary results showed a significant correlation between DNA 
Table 3 Multivariate analysis in the combined cohort $(N=378)$

\begin{tabular}{lccc}
\hline Characteristic & $\boldsymbol{P}$ value & $\begin{array}{c}\text { Hazard } \\
\text { ratio }\end{array}$ & $\begin{array}{c}\mathbf{9 5 \%} \mathrm{Cl} \text { for hazard } \\
\text { ratios }\end{array}$ \\
\hline $\begin{array}{l}\text { Tumor type } \\
\quad \text { Luminal } \\
\text { (reference) }\end{array}$ & 0.09 & & \\
$\quad$ Basal & 0.02 & 4.04 & 1.3 to 12.8 \\
HER2 & 0.15 & 1.64 & 0.8 to 3.2 \\
$\quad$ Null & 0.67 & 1.23 & 0.5 to 3.2 \\
Grade & & & \\
1 & 0.000 & & 1.2 to 5.8 \\
2 & 0.02 & 2.61 & 5.2 to 31.4 \\
3 & 0.0001 & 12.72 & 0.4 to 1.3 \\
Lymph node status & 0.28 & 0.75 & 0.4 to 1.2 \\
Tumor size $>20$ mm & 0.18 & 0.69 & \\
\hline
\end{tabular}

Multivariate analysis using a binary logistic regression model of the effect of $\mathrm{SIAH} 2$ expression on tumor subtype (with luminal tumors as a reference), tumor grade, lymph node status and tumor size.

${ }^{\text {aa }} \mathrm{SIAH} 2$, seven in absentia homolog 2 gene.

95\% Cl, 95\% confidence interval; HER2, human epidermal growth factor receptor 2 .

copy number and mRNA expression, supporting this hypothesis. Specifically, we found that basal-like tumors showed copy number gain of the SIAH2 locus more frequently than luminal tumors and that basal-like tumors containing copy number gain were associated with high expression of SIAH2. Nevertheless, using a more sensitive and specific method for quantifying gene copy number such as fluorescence in situ hybridization assay, together with SIAH2 protein expression in a validation cohort, would be of interest to confirm this finding and assess whether true amplification occurs.

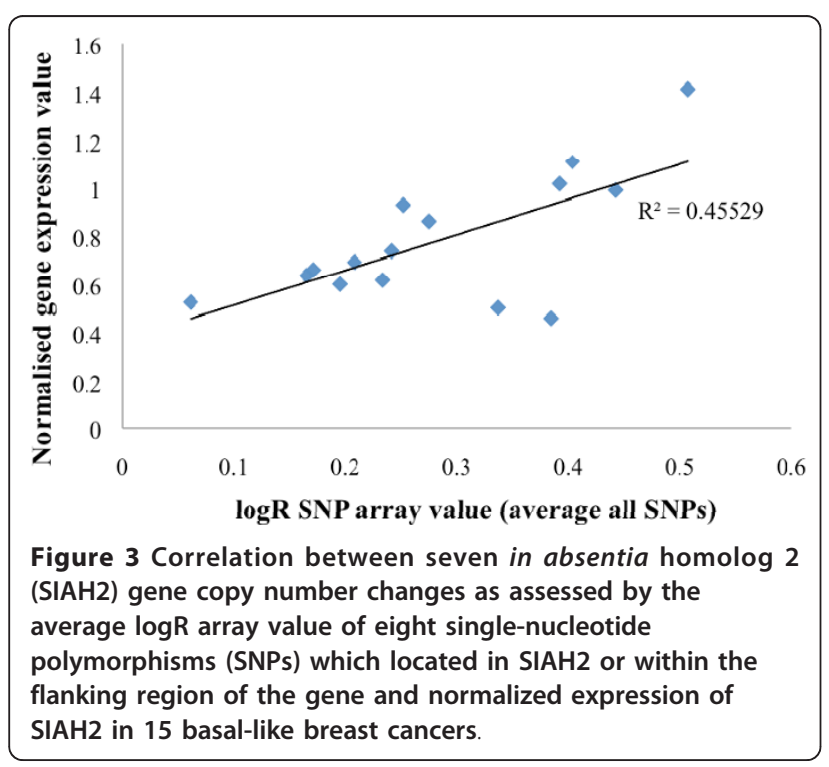

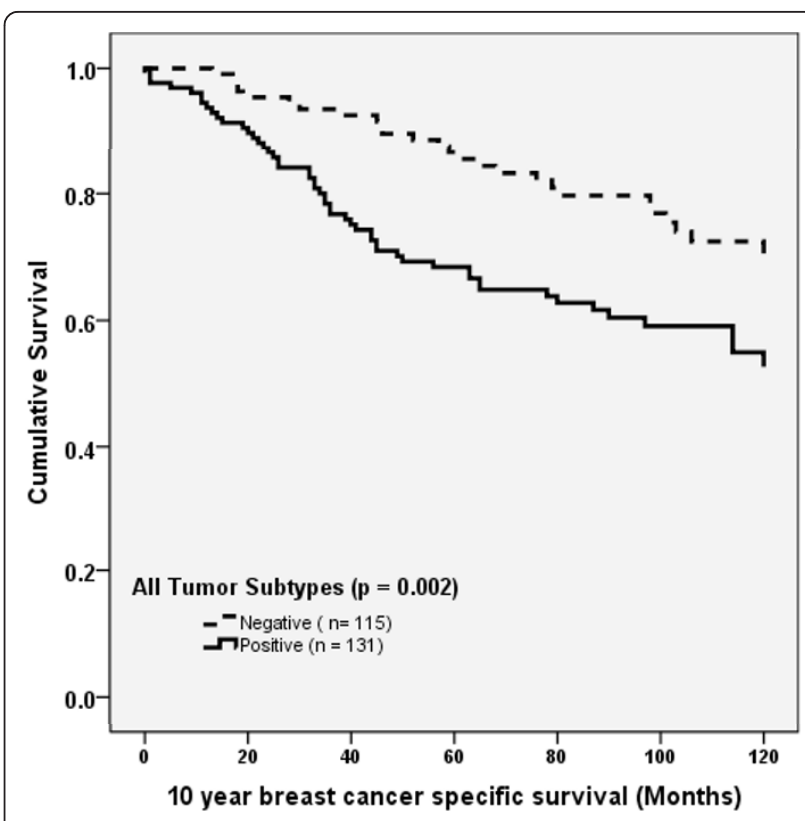

Figure 4 Kaplan-Meier curves stratified by seven in absentia homolog 2 (SIAH2) gene expression for relapse-free survival in (A) all tumors $(N=246)$ and (B) luminal tumors $(n=144)$.

Although we observed a significantly shorter relapsefree survival in patients with SIAH2-positive tumors in univariate analysis, this was not confirmed in the multivariate analysis model that included conventional prognostic factors such as tumor size, tumor grade and lymph node status. While SIAH2 was not an independent survival factor in a multivariate analysis model, it was prognostic in the univariate analysis because of its strong association with the basal-like phenotype, which is an independent prognostic factor. Even so, the role of

Table 4 Multivariate analysis using the Cox regression model of relapse-free survival in all breast cancers of validation cohort $(N=245)^{a}$

\begin{tabular}{lccc}
\hline Characteristic & $\boldsymbol{P}$ value & Hazard ratio & $\mathbf{9 5 \%} \mathbf{C l}$ for hazard ratios \\
\hline SIAH2 & 0.47 & 1.24 & 0.7 to 2.2 \\
Grade & & & \\
$\quad 1$ (reference) & 0.54 & & \\
$\quad 2$ & 0.70 & 0.83 & 0.3 to 2.1 \\
$\quad 3$ & 0.70 & 1.22 & 0.4 to 3.3 \\
Tumor size & 0.12 & 1.37 & 0.9 to 2.2 \\
Lymph node status & 0.001 & 2.25 & 1.4 to 3.7 \\
Tumor type & & & \\
$\quad$ Luminal & 0.004 & & \\
$\quad$ Basal-like & 0.005 & 3.0 & 1.4 to 6.4 \\
$\quad$ HER2 & 0.001 & 2.9 & 1.6 to 5.7 \\
$\quad$ Null & 0.016 & 2.5 & 1.2 to 5.1 \\
\hline
\end{tabular}

${ }^{\mathrm{a}} \mathrm{SIAH} 2$, seven in absentia homolog 2 gene.

$95 \% \mathrm{Cl}, 95 \%$ confidence interval, HER2, human epidermal growth factor receptor 2. 
SIAH2 remains unclear. Thus a report has suggested that patients with SIAH2-positive, ER-positive tumors have a significantly longer progression-free survival than patients with SIAH2-negative tumors and also that Siah2 levels might be a predictive marker of estrogenresponsive disease [28]. The discrepancy between these findings and our own are likely due to the use of mRNA levels to measure SIAH2 by Jansen et al. [28], and, despite enriching for neoplastic cells, the stromal compartment contributed to the overexpression of SIAH2, thus confounding the comparison. In support of this notion, it has been shown by expression microarrays that downregulated SIAH2 in brain metastasis of breast cancer corresponds with low stromal contamination [29]. The concept of SIAH2 being a good prognostic and/or predictive parameter is not in accord with the role of SIAH2 in regulating the hypoxic response or with the observation that inhibition of SIAH2 is associated with reduced metastases in animal models [30]. SIAH2 appears to have several mechanisms of mediating its effect. Some SIAH2 substrates bind directly through an AXVXP motif, some require adaptor proteins and still others are targeted independently of the above sequence motif [3]. Thus, depending on the cell context, SIAH2 is likely to have a variety of effects.

SIAH2 is critical to the level of the hypoxic response and therefore is a potential target for anticancer therapy. Indeed, since HIF activation results in the regulation of a large number of genes, interference with this pathway would have broad antineoplastic effects in contrast to targeting individual genes, such as VEGF, with bevacizumab, which is currently used in the clinic. Indeed, menadione, a specific inhibitor of SIAH2, increased expression of prolyl hydroxylase with a concomitant decrease in levels of HIF-1 $\alpha$. This promising therapeutic approach also retarded the growth of melanoma xenografts [31]. The potential of this approach has also been investigated using a short protein fragment that competitively binds to Siah, resulting in reduced breast cancer growth, which appeared to be mediated through inhibition of the hypoxic response [4].

\section{Conclusions}

In summary, we have shown that in situ and invasive breast carcinomas upregulate SIAH2 and that it is preferentially highly expressed in the basal-like subtype, which can be accounted for in part by increased gene copy number. High levels of SIAH2 may be partly responsible for the enhanced hypoxic drive that underlies this tumor type, which is chemotherapy- and radiotherapy-resistant. Targeting SIAH2, the most apical regulator identified in the hypoxic response pathway, may be a suitable option for anticancer therapy in this breast tumor subtype.

\section{Additional material}

\author{
Additional file 1: Supplementary Table 1. Flow of breast cancer \\ patients through the study, according to REMARK criteria [7] \\ Additional file 2: Supplementary Table 2. Tumor phenotype of 60 \\ samples for which seven in absentia homolog 2 (SIAH2) gene \\ methylation analysis was performed
}

Additional file 3: Supplementary Table 3. Contingency table of ductal carcinoma in situ (DCIS) and available clinicopathological variables

Additional file 4: Supplementary Table 4. Contingency table of seven in absentia homolog 2 (SIAH2) gene expression in invasive breast carcinomas of the primary cohort with clinicopathological parameters

Additional file 5: Supplementary Table 5. Contingency table of seven in absentia homolog 2 (SIAH2) gene expression in invasive breast carcinomas of the initial cohort with clinicopathological parameters

Additional file 6: Supplementary Figure 1. Seven in absentia homolog 2 (SIAH2) gene methylation in breast carcinoma samples. Methylationsensitive high-resolution melting (MS-HRM) detects sample methylation status by melting the amplicons after polymerase chain reaction assay. Methylated samples melt later than unmethylated samples, as they have cytosines in their sequences rather than the thymines after the bisulfite modification. Two estrogen receptor (ER)-positive and two ER-negative breast carcinomas show no methylation in SIAH2. Standard controls of $100 \%, 50 \%, 10 \%$ and 0\% methylation are shown. The curve for each sample represents data from duplicate samples.

\section{Abbreviations}

ER: estrogen receptor; HIF: hypoxia-inducible factor; PR: progesterone receptor; Siah: seven in absentia homologue.

\section{Acknowledgements}

This study was supported by the Victorian Breast Cancer Research Consortium, the National Health and Medical Research Council (grant 481378 (to SAO), grant 427601 (to RLS) and grant 535947 (SAO), the Association for Cancer Research UK (grant AICR 09-0676 (to AM)), Cancer Council Victoria (grant to DDB and AM) the Cancer Institute NSW (grant 07/ CRF/1-06 (to SAO) and grant 07-CDF-1/28 (EAM), the Australian Cancer Research Foundation (ACRF), the RT Hall Trust and the Petre Foundation, Australia. We thank Professor Adrian L Harris for the kind donation of the breast tumor DNA for methylation analysis.

\section{Author details}

${ }^{1}$ Department of Pathology, Peter MacCallum Cancer Centre, Locked Bag 1 A'Beckett Street, Melbourne, Victoria 8006, Australia. ${ }^{2}$ Research Division, Cancer Genetics and Genomics, Peter MacCallum Cancer Centre, Locked Bag 1 A'Beckett Street, Melbourne, Victoria 8006, Australia. ${ }^{3}$ Department of Pathology, University of Melbourne, Royal Parade, Parkville, Victoria 3010, Australia. ${ }^{4}$ Queensland Centre for Medical Genomics, Institute for Molecular Bioscience, University of Queensland, Brisbane, Queensland 4072, Australia.

${ }^{5}$ Cancer Research Program, Garvan Institute of Medical Research, 384 Victoria Street, Darlinghurst, NSW 2010, Australia. ${ }^{6}$ South East Area Laboratory Services, St George Hospital, Kogarah, NSW 2217, Australia. ${ }^{7}$ School of Medicine, University of Western Sydney, Campbelltown NSW 2751, Australia. ${ }^{8}$ School of Medical Sciences, Faculty of Medicine, University of New South Wales, Sydney, NSW 2052, Australia. 'Department of Tissue Pathology and Diagnostic Oncology, Royal Prince Alfred Hospital, Camperdown, NSW 2006, Australia. ${ }^{10}$ Faculty of Medicine, University of Sydney, NSW 2006, Australia. ${ }^{11}$ Department of Medical Oncology \& Breast Cancer Institute of New South Wales, University of Sydney, Westmead Hospital, Westmead, NSW 2145, Australia. ${ }^{12}$ Western Clinical School, University of Sydney, Westmead Hospital, Westmead, NSW 2145, Australia.

\section{Authors' contributions}

PC carried out the scoring of the tissue microarrays (TMAs) and calculated statistics. AM conceived and designed the study and drafted the manuscript. MCPL and JES stained the TMAs. CSFW assisted in staining the TMAs and drafting the manuscript. NW conducted copy number experiments and analysis. KTH conducted the promoter analysis. AD conducted and 
supervised promoter analysis. EKAM and SAO generated TMAs and scored correlative markers. CMM conducted clinical follow-up. RLS provided TMAs, helped in drafting the manuscript and provided critical discussions. DB helped in designing the study. SBF scored the TMAs, conceived and designed the study and drafted the manuscript. All authors read and approved the final version of the manuscript.

\section{Competing interests}

The authors declare that they have no competing interests.

Received: 17 September 2010 Revised: 23 December 2010

Accepted: 9 February 2011 Published: 9 February 2011

\section{References}

1. Harris AL: Hypoxia: a key regulatory factor in tumour growth. Nat Rev Cancer 2002, 2:38-47.

2. House CM, Moller A, Bowtell DD: Siah proteins: novel drug targets in the Ras and hypoxia pathways. Cancer Res 2009, 69:8835-8838.

3. Nakayama K, Qi J, Ronai Z: The ubiquitin ligase Siah2 and the hypoxia response. Mol Cancer Res 2009, 7:443-451.

4. Moller A, House CM, Wong CS, Scanlon DB, Liu MC, Ronai Z, Bowtell DD: Inhibition of Siah ubiquitin ligase function. Oncogene 2009, 28:289-296.

5. Bos R, Zhong H, Hanrahan CF, Mommers EC, Semenza GL, Pinedo HM, Abeloff MD, Simons JW, van Diest PJ, van der Wall E: Levels of hypoxiainducible factor-1a during breast carcinogenesis. J Natl Cancer Inst 2001, 93:309-314.

6. Dales JP, Garcia S, Meunier-Carpentier S, Andrac-Meyer L, Haddad O, Lavaut MN, Allasia C, Bonnier P, Charpin C: Overexpression of hypoxiainducible factor HIF-1a predicts early relapse in breast cancer: retrospective study in a series of 745 patients. Int J Cancer 2005, 116:734-739.

7. Tan EY, Yan M, Campo L, Han C, Takano E, Turley H, Candiloro I, Pezzella F, Gatter KC, Millar EK, O'Toole SA, McNeil CM, Crea P, Segara D,

Sutherland RL, Harris AL, Fox SB: The key hypoxia regulated gene CAIX is upregulated in basal-like breast tumours and is associated with resistance to chemotherapy. Br J Cancer 2009, 100:405-411.

8. Millar EK, Anderson LR, McNeil CM, O'Toole SA, Pinese M, Crea P, Morey AL, Biankin AV, Henshall SM, Musgrove EA, Sutherland RL, Butt AJ: BAG-1 predicts patient outcome and tamoxifen responsiveness in ER-positive invasive ductal carcinoma of the breast. Br J Cancer 2009, 100:123-133.

9. Schmidt RL, Park CH, Ahmed AU, Gundelach JH, Reed NR, Cheng S, Knudsen BE, Tang AH: Inhibition of RAS-mediated transformation and tumorigenesis by targeting the downstream E3 ubiquitin ligase seven in absentia homologue. Cancer Res 2007, 67:11798-11810.

10. Ahmed AU, Schmidt RL, Park CH, Reed NR, Hesse SE, Thomas CF, Molina JR, Deschamps C, Yang P, Aubry MC, Tang AH: Effect of disrupting seven-in-absentia homolog 2 function on lung cancer cell growth. J Natl Cancer Inst 2008, 100:1606-1629.

11. Tan EY, Campo L, Han C, Turley H, Pezzella F, Gatter KC, Harris AL, Fox SB: Cytoplasmic location of factor-inhibiting hypoxia-inducible factor is associated with an enhanced hypoxic response and a shorter survival in invasive breast cancer. Breast Cancer Res 2007, 9:R89.

12. Mirzoeva OK, Das D, Heiser LM, Bhattacharya S, Siwak D, Gendelman R, Bayani N, Wang NJ, Neve RM, Guan Y, Hu Z, Knight Z, Feiler HS, Gascard P, Parvin B, Spellman PT, Shokat KM, Wyrobek AJ, Bissell MJ, McCormick F, Kuo WL, Mills GB, Gray JW, Korn WM: Basal subtype and MAPK/ERK kinase (MEK)-phosphoinositide 3-kinase feedback signaling determine susceptibility of breast cancer cells to MEK inhibition. Cancer Res 2009, 69:565-572.

13. Nielsen TO, Hsu FD, Jensen K, Cheang M, Karaca G, Hu Z, HernandezBoussard T, Livasy C, Cowan D, Dressler L, Akslen LA, Ragaz J, Gown AM, Gilks CB, van de Rijn M, Perou CM: Immunohistochemical and clinical characterization of the basal-like subtype of invasive breast carcinoma. Clin Cancer Res 2004, 10:5367-5374.

14. Wojdacz TK, Dobrovic A: Methylation-sensitive high resolution melting (MS-HRM): a new approach for sensitive and high-throughput assessment of methylation. Nucleic Acids Res 2007, 35:e41.

15. Waddell N, Arnold J, Cocciardi S, da Silva L, Marsh A, Riley J, Johnstone CN, Orloff M, Assie G, Eng C, Reid L, Keith P, Yan M, Fox S, Devilee P, Godwin AK, Hogervorst FB, Couch F, Investigators KConFab, Grimmond S,
Flanagan JM, Khanna K, Simpson PT, Lakhani SR, Chenevix-Trench G: Subtypes of familial breast tumours revealed by expression and copy number profiling. Breast Cancer Res Treat 2010, 123:661-677.

16. Fukuba H, Yamashita H, Nagano $Y$, Jin HG, Hiji M, Ohtsuki T, Takahashi T, Kohriyama T, Matsumoto M: Siah-1 facilitates ubiquitination and degradation of factor inhibiting HIF-1a (FIH). Biochem Biophys Res Commun 2007, 353:324-329.

17. Simon MC: Siah proteins, HIF prolyl hydroxylases, and the physiological response to hypoxia. Cell 2004, 117:851-853.

18. Della NG, Senior PV, Bowtell DD: Isolation and characterisation of murine homologues of the Drosophila seven in absentia gene (sina). Development 1993, 117:1333-1343.

19. Frasor J, Danes JM, Funk CC, Katzenellenbogen BS: Estrogen downregulation of the corepressor $\mathrm{N}-\mathrm{CoR}$ : mechanism and implications for estrogen derepression of N-CoR-regulated genes. Proc Natl Acad Sci USA 2005, 102:13153-13157.

20. Chin K, DeVries S, Fridlyand J, Spellman PT, Roydasgupta R, Kuo WL, Lapuk A, Neve RM, Qian Z, Ryder T, Chen F, Feiler H, Tokuyasu T, Kingsley C, Dairkee S, Meng Z, Chew K, Pinkel D, Jain A, Ljung BM, Esserman L, Albertson DG, Waldman FM, Gray JW: Genomic and transcriptional aberrations linked to breast cancer pathophysiologies. Cancer Cell 2006, 10:529-541.

21. Desmedt C, Piette F, Loi S, Wang Y, Lallemand F, Haibe-Kains B, Viale G, Delorenzi M, Zhang Y, d'Assignies MS, Bergh J, Lidereau R, Ellis P, Harris AL, Klijn JG, Foekens JA, Cardoso F, Piccart MJ, Buyse M, Sotiriou C: Strong time dependence of the 76-gene prognostic signature for node-negative breast cancer patients in the TRANSBIG multicenter independent validation series. Clin Cancer Res 2007, 13:3207-3214.

22. Hess KR, Anderson K, Symmans WF, Valero V, Ibrahim N, Mejia JA, Booser D, Theriault RL, Buzdar AU, Dempsey PJ, Rouzier R, Sneige N, Ross JS, Vidaurre T, Gomez HL, Hortobagyi GN, Pusztai L: Pharmacogenomic predictor of sensitivity to preoperative chemotherapy with paclitaxel and fluorouracil, doxorubicin, and cyclophosphamide in breast cancer. $J$ Clin Oncol 2006, 24:4236-4244.

23. Miller LD, Smeds J, George J, Vega VB, Vergara L, Ploner A, Pawitan Y, Hall P, Klaar S, Liu ET, Bergh J: An expression signature for p53 status in human breast cancer predicts mutation status, transcriptional effects, and patient survival. Proc Natl Acad Sci USA 2005, 102:13550-13555.

24. Minn AJ, Gupta GP, Siegel PM, Bos PD, Shu W, Giri DD, Viale A, Olshen AB, Gerald WL, Massague J: Genes that mediate breast cancer metastasis to lung. Nature 2005, 436:518-524.

25. Wang $Y$, Klijn JG, Zhang Y, Sieuwerts AM, Look MP, Yang F, Talantov D, Timmermans M, Meijer-van Gelder ME, Yu J, Jatkoe T, Berns EM, Atkins D, Foekens JA: Gene-expression profiles to predict distant metastasis of lymph-node-negative primary breast cancer. Lancet 2005, 365:671-679.

26. Manié E, Vincent-Salomon A, Lehmann-Che J, Pierron G, Turpin E, Warcoin M, Gruel N, Lebigot I, Sastre-Garau X, Lidereau R, Remenieras A, Feunteun J, Delattre O, de Thé H, Stoppa-Lyonnet D, Stern MH: High frequency of TP53 mutation in BRCA1 and sporadic basal-like carcinomas but not in BRCA1 luminal breast tumors. Cancer Res 2009, 69:663-671.

27. Gatza ML, Lucas JE, Barry WT, Kim JW, Wang Q, Crawford MD, Datto MB, Kelley M, Mathey-Prevot B, Potti A, Nevins JR: A pathway-based classification of human breast cancer. Proc Natl Acad Sci USA 2010, 107:6994-6999.

28. Jansen MP, Ruigrok-Ritstier K, Dorssers LC, van Staveren IL, Look MP, Meijervan Gelder ME, Sieuwerts AM, Helleman J, Sleijfer S, Klijn JG, Foekens JA, Berns EM: Downregulation of SIAH2, an ubiquitin E3 ligase, is associated with resistance to endocrine therapy in breast cancer. Breast Cancer Res Treat 2009, 116:263-271.

29. Palmieri D, Fitzgerald D, Shreeve SM, Hua E, Bronder JL, Weil RJ, Davis S, Stark AM, Merino MJ, Kurek R, Mehdorn HM, Davis G, Steinberg SM, Meltzer PS, Aldape K, Steeg PS: Analyses of resected human brain metastases of breast cancer reveal the association between upregulation of hexokinase 2 and poor prognosis. Mol Cancer Res 2009, 7:1438-1445

30. Qi J, Nakayama K, Gaitonde S, Goydos JS, Krajewski S, Eroshkin A, BarSagi $D$, Bowtell $D$, Ronai Z: The ubiquitin ligase Siah2 regulates tumorigenesis and metastasis by HIF-dependent and -independent pathways. Proc Natl Acad Sci USA 2008, 105:16713-16718. 
31. Shah M, Stebbins JL, Dewing A, Qi J, Pellecchia M, Ronai ZA: Inhibition of Siah2 ubiquitin ligase by vitamin $K_{3}$ (menadione) attenuates hypoxia and MAPK signaling and blocks melanoma tumorigenesis. Pigment Cell Melanoma Res 2009, 22:799-808.

doi:10.1186/bcr2828

Cite this article as: Chan et al:: The expression of the ubiquitin ligase SIAH2 (seven in absentia homolog 2) is mediated through gene copy number in breast cancer and is associated with a basal-like phenotype and p53 expression. Breast Cancer Research 2011 13:R19.

Submit your next manuscript to BioMed Central and take full advantage of:

- Convenient online submission

- Thorough peer review

- No space constraints or color figure charges

- Immediate publication on acceptance

- Inclusion in PubMed, CAS, Scopus and Google Scholar

- Research which is freely available for redistribution

Submit your manuscript at www.biomedcentral.com/submit 